Documentation et bibliothèques

DOCUMENTATION BIBLIOTHEQUES

\title{
Présent et futur du réseau des bibliothèques publiques au Québec
}

\section{Yvon-André Lacroix}

Volume 25, numéro 4, décembre 1979

URI : https://id.erudit.org/iderudit/1054292ar

DOI : https://doi.org/10.7202/1054292ar

Aller au sommaire du numéro

\section{Éditeur(s)}

Association pour l'avancement des sciences et des techniques de la documentation (ASTED)

\section{ISSN}

0315-2340 (imprimé)

2291-8949 (numérique)

Découvrir la revue

\section{Citer cet article}

Lacroix, Y.-A. (1979). Présent et futur du réseau des bibliothèques publiques au Québec. Documentation et bibliothèques, 25(4), 177-188.

https://doi.org/10.7202/1054292ar

\section{Résumé de l'article}

L'auteur dresse d'abord un tableau détaillé de la situation présente des bibliothèques publiques au Québec. Il expose à l'aide des données statistiques appropriées l'écart entre les normes établies par le Service des bibliothèques publiques du ministère des Affaires culturelles et les réalisations des bibliothèques. Il fait ensuite ressortir la nécessité de changer le statut des bibliothèques à l'intérieur des services municipaux pour en faire un service autonome et il démontre que cette amélioration passe par un certain lobbying auprès des élus. Il conclut en recommandant quelques mesures concrètes devant aboutir à une meilleure connaissance de la bibliothèque publique par les citoyens.
Tous droits réservés (C) Association pour l'avancement des sciences et des techniques de la documentation (ASTED), 1979
Ce document est protégé par la loi sur le droit d'auteur. L'utilisation des services d'Érudit (y compris la reproduction) est assujettie à sa politique d'utilisation que vous pouvez consulter en ligne.

https://apropos.erudit.org/fr/usagers/politique-dutilisation/ 


\title{
Présent et futur du réseau des bibliothèques publiques au Québec
}

\author{
Yvon-André Lacroix \\ Directeur \\ Service de la Bibliothèque municipale de Brossard
}

L'auteur dresse d'abord un tableau détaillé de la situation présente des bibliothèques publiques au Québec. II expose à l'aide des données statistiques appropriées l'écart entre les normes établies par le Service des bibliothèques publiques du ministère des Affaires culturelles et les réalisations des bibliothèques. II fait ensuite ressortir la nécessité de changer le statut des bibliothèques à l'intérieur des services municipaux pour en faire un service autonome et il démontre que cette amélioration passe par un certain lobbying auprès des élus. II conclut en recommandant quelques mesures concrètes devant aboutir à une meilleure connaissance de la bibliothèque publique par les citoyens.

The author begins by giving us a detailed picture of the present situation of public libraries. With the help of the appropriate statistical data, he demonstrates the wide margin which exists between the libraries as they are and the standards established by the "Service des bibliothèques publiques" of the "ministère des Affaires culturelles». He then demonstrates the need to change the status of the library within the municipal framework in order to make it autonomous, a change which can only be accomplished by lobbying. He concludes by recommending certain concrete measures which should result in a better knowledge of public libraries by the public.

El autor empieza describiendo con todo detalle ia situación de las bibliotecas públicas en la provincia de Quebec. Basándose en datos estadísticos apropiados, explica la diferencia entre las normas establecidas por el "Service des bibliothèques publiques" del "ministère des Affaires culturelles» y las realizaciones de las bibliotecas. Destaca la necesidad de cambiar el estatuto de las bibliotecas en los servicios municipales y de formar un servicio autónomo para éstas, y demuestra que una mejora de este tipo necesita una especie de "lobbying" con el gobierno. Concluye recomendando algunas medidas concretas que deberían llegar a un mejor conocimiento de la biblioteca pública por los ciudadanos.

N.B.: L'auteur ayant déposé son texte en août 1979, il n'a pu faire valoir comme il l'aurait souhaité les espoirs suscités par le document du ministre Plan quinquennal de développement (1980-84) des bibliothèques publiques dans les municipalités de 5.000 habitants et p̀lus. 
Le but de cet article n'est pas de tracer un tableau systématique et complet du dossier des bibliothèques publiques. Cela pourrait faire l'objet de plusieurs mémoires et thèses. II s'agit plutôt d'en analyser la performance en fonction de certains critères dits minima pouvant servir à leur évaluation et à l'établissement des priorités définies par les bibliothèques publiques subventionnées dans leur plan quinquennal déposé en décembre 1978.

Ces critères ne sont évidemment pas définitifs et méritent réflexion avant de le devenir. Ils peuvent néanmoins servir à engager une discussion, nécessaire et indispensable, entre les partis concernés.

En dernière partie, quelques problèmes, parmi tant d'autres, seront brièvement exposés.

\section{Tableau de la situation et critères minima}

\section{Les bibliothèques et l'aide gouvernementale}

C'est avec enthousiasme et satisfaction que les directeurs et le personnel des bibliothèques publiques ont accueilli les multiples déclarations du ministre des Affaires culturelles, M. Denis Vaugeois, à savoir que la priorité numéro un de son ministère serait dorénavant donnée aux bibliothèques publiques '. De grands espoirs d'amélioration substantielle du réseau des bibliothèques publiques sont désormais permis.

Depuis 1959, année de la création du Service des bibliothèques publiques, le Gouvernement s'occupe des bibliothèques publiques par l'établissement de normes de fonctionnement, de subventions et d'aide technique. En 1979, les argents fournis par le ministère des Affaires culturelles comptent pour $23.9 \%$ des dépenses totales de fonctionnement des bibliothèques publiques subventionnées.

Avec le nouveau plan de réforme de la fiscalité municipale, la majorité des subventions aux municipalités sont abolies, à l'exception de celles qui s'appliquent aux bibliothèques publiques.

En 1979, une bibliothèque est éligible aux subventions provinciales si, entre autres conditions, elle reçoit de la municipalité qu'elle dessert $0.03 \$$ par $100 \$$ d'évaluation foncière imposable rajustée à $100 \%$ de la valeur réelle (ce montant sera haussé à $0.05 \$$ en 1983 ). Évidemment, cela est peu ou beaucoup, selon l'état de richesse d'une municipalité. Pour corriger cette injustice, il y a des maxima gradués selon l'évaluation foncière imposable par tête: ainsi une ville dont l'évaluation dépasse $8,700 \$$ par tête ne reçoit pas plus de $1.65 \$$ de subvention par habitant et celle dont l'évaluation est inférieure à $4,350 \$$ reçoit $3.30 \$$.

A ces subventions statutaires s'ajoutent l'aide à l'animation et

1. Discours du ministre des Affaires culturelles, M. Denis Vaugeois, au $5^{e}$ congrès de I'ASTED, le 25 octobre 1978. 
l'aide pour l'embauche de bibliothécaires professionnels: $6,000 \$$ par an pendant trois ans pour chaque nouveau bibliothécaire employé.

D'autres programmes d'aide sont prévus: aide à l'achat de livres, "aide à la construction ou à l'agrandissement des bibliothèques dans les municipalités où le rapport entre la superficie disponible et la population est trop faible. ${ }^{2}$

En 1979, 89 municipalités (75 ont plus de 5,000 habitants) ont eu droit aux subventions provinciales. Seize autres municipalités ont une bibliothèque, mais leur contribution est inférieure au minimum requis et elles se voient ainsi refuser une subvention. Cinq bibliothèques ont cessé d'être subventionnées en janvier 1979, faute de respecter le minimum de $0.03 \$$ par $100 \$$ d'évaluation foncière. De plus, neuf municipalités de plus de 5,000 habitants sont affiliées à une Bibliothèque Centrale de Prêt (B.C.P.). II reste cependant le nombre impressionnant de 52 sur 155 municipalités de plus de 5,000 habitants (et non les moindres, Charlesbourg par exemple avec 65,000 habitants, Beauport avec 56,000 habitants...) et 940 municipalités de moins de 5,000 habitants sans service de bibliothèque reconnu, soit $1,739,675$ habitants $(27.6 \%)$.

Fort heureusement, depuis les cinq dernières années, le milieu des bibliothèques publiques est en expansion grâce aux efforts multiples déployés par le ministère des Affaires culturelles, les différentes administrations municipales et les nouveaux directeurs chargés d'ériger de toute pièce de nouvelles bibliothèques. Ainsi depuis trois ans, seize nouvelles bibliothèques ont été créées au Québec et, dans plusieurs cas, avec tout l'argent nécessaire. Sur la Rive-Sud de Montréal, il y a eu depuis trois ans un essor phénoménal: déblocage de crédits pour construction, aménagement, embauche de bibliothécaires (on y comptait trois bibliothécaires, il y en a maintenant douze) et développement des collections.

Dans presque chacune des régions du Québec, il y a actuellement au moins une bonne bibliothèque municipale dont l'essor peut avoir un certain effet d'entraînement sur le développement des autres. Parallèlement à cet effort, le ministère des Affaires culturelles a favorisé la création de cinq nouvelles B.C.P. depuis 1975. Ainsi les huit B.C.P. actuelles desservent l'ensemble des régions et des municipalités de moins de 5,000 habitants.

A part cette aide financière importante, les directeurs des bibliothèques publiques sont encore pour le moment très isolés, ne jouissant d'aucune expertise technique susceptible de les aider à aménager, construire, administrer, rationaliser, informatiser. II n'y a pas de coordination véritable des efforts en vue d'aplanir les difficultés abordées ci-après et le «laisser-faire culturel» des municipalités vis-à-vis des bibliothèques est toujours de mise.

\section{Population par bibliothécaire}

Actuellement, le ministère des Affaires culturelles accorde une subvention annuelle de $6,000 \$$ pour chaque bibliothécaire embauché

2. Québec, Ministère d'État au Développement culturel, La politique québécoise du développement culturel, Québec, Editeur officiel, 1978, vol. 2, p. 393. 
par une ville dont le nombre de bibliothécaires est inférieur à 1 par 12,000 habitants $^{3}$. Ce programme est évidemment offert pour encourager les municipalités à embaucher des bibliothécaires professionnels. Cette subvention n'est attribuée que durant les trois premières années d'emploi de ce bibliothécaire professionnel ${ }^{4}$.

En 1978, il y avait 131 bibliothécaires embauchés par 75 municipalités de 5,000 habitants et plus: on retrouvait 52 de ces bibliothécaires à la Ville de Montréal. Le tableau 1 présente une synthèse de la répartition des bibliothécaires.

Tableau 1:

Bibliothécaires dans les municipalités de 5,000 habitants et plus

Bibliothèques subventionnées

avec un seul bibliothécaire

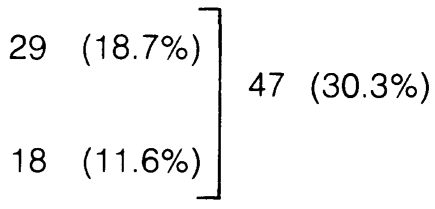

Bibliothèques subventionnées

avec 2 bibliothécaires et plus

Bibliothèques subventionnées

sans bibliothécaire

$28(18.1 \%)$

Autres bibliothèques municipales

sans bibliothécaire

$25(16.1 \%)$

Municipalités de plus de 5,000

habitants sans bibliothèque

Sur un potentiel de 155 bibliothèques publiques, on ne compte que 47 villes où l'on trouve un bibliothécaire. Et les chiffres vont de zéro bibliothécaire à Chicoutimi (58,800 habitants) et un seul à Montréal-Nord (97,300 habitants) à cinq à Westmount (22,300 habitants). Les meilleures performances en ce domaine sont les suivantes:

$\begin{array}{ll}\text { Westmount: } & 5 \text { bibliothécaires pour } 22,300, \\ & \text { soit } 1 \text { pour } 4,460 \text { habitants } \\ \text { Ville Mont-Royal: } & 4 \text { bibliothécaires pour } 20,500, \\ & \text { soit } 1 \text { pour } 5,125 \text { habitants } \\ \text { Pointe-Claire: } & 5 \text { bibliothécaires pour } 25,900, \\ & \text { soit } 1 \text { pour } 5,180 \text { habitants }\end{array}$

3. Pour bien fonctionner et donner tous les services, une bibliothèque doit disposer de 1 bibliothécaire par 6,000 habitants, selon les normes du ministère des Affaires culturelles.

4. II faudrait peut-être trouver un autre mécanisme pour cette aide, puisque plusieurs hauts fonctionnaires municipaux peuvent, grâce à ce $6,000 \$$, dire qu'un bibliothécaire leur coûte le salaire d'une secrétaire. Cette sorte de main-d'oeuvre professionnelle et qualifiée, mais à bon marché, ne rehausse pas le prestige du bibliothécaire dans son milieu. Pour ces fonctionnaires comme pour bien d'autres personnes, un professionnel n'est jamais subventionné.

5. Québec, Ministère des Affaires culturelles, Service des bibliothèques publiques, Biblio-contact, vol. 5, no 1 (printemps 1979). 
Il y a donc au Québec 131 bibliothécaires qui desservent $4,611,329$ habitants, soit 1 par 35,000 habitants ou près de $600 \%$ en-dessous de la norme de 1 bibliothécaire pour 6,000 habitants. A part les trois municipalités citées plus haut, aucune ne rencontre cette norme. En rêvant en couleur, on peut se dire qu'il existe là un marché potentiel pour 637 nouveaux postes de bibliothécaires professionnels. Rêver? L'Ontario avait 943 bibliothécaires travaillant à temps plein dans ses bibliothèques publiques en 1976 (1 pour 8,763 habitants).

\section{Dépenses des bibliothèques par habitant}

En premier lieu, il faut préciser que nous estimons le seuil critique au-dessous duquel une bibliothèque publique vivote, sinon régresse, à un montant de $5 \$$, montant que la municipalité devrait consacrer à son service de bibliothèque. Examinons les tableaux suivants tirés des prévisions budgétaires 1978 et 1979 des bibliothèques subventionnées ${ }^{6}$.

Tableau 2:

Dépenses des bibliothèques publiques par habitant

$\begin{array}{cc}\text { Prévisions } & \text { Prévisions } \\ \text { budgétaires } & \text { budgétaires } \\ 1978 & 1979\end{array}$

Nombre de bibliothèques subventionnées ${ }^{7}$

$94(100 \%)$

$89(100 \%)$

Bibliothèques consacrant moins de $5 \$ /$ tête

$66(70 \%)$

$37(42 \%)$

Bibliothèques consacrant entre $5 \$$ et $9.99 \$ /$ tête

$19(20 \%)$

$41(46 \%)$

Bibliothèques consacrant plus de $10 \$$

$9(10 \%)$

$11(12 \%)$

Donc, seulement 28 (30\%) en 1978 et 52 (58\%) en 1979 sont au-dessus du seuil de la pauvreté. Même celles qui se situent entre $5 \$$ et $9.99 \$$ /tête doivent faire des miracles pour n'être que très relativement efficaces aux yeux de leur clientèle respective. Au Québec, actuellement, une bibliothèque publique qualifiée de très riche est celle qui peut disposer de plus de $10 \$$ par habitant: $9(10 \%)$ en 1978 et $11(12 \%)$ en 1979 (les deux nouvelles sont celles de Saint-Laurent et de Beaconsfield). Parmi celles-ci, six sont en très grande majorité anglophones (Pointe-Claire, Ville Mont-Royal, Westmount, Côte St-Luc, Dorval et Beaconsfield), une, Saint-Laurent, a une population dont une moitié est anglophone et l'autre francophone, et les quatre autres (Montréal-Est, Marieville, St-Eustache et Sept-lles) sont à majorité francophone.

7. Comprend aussi des municipalités de 5,000 habitants et moins. 
Nombre de livres par habitant

Pour mesurer l'état de richesse ou de pauvreté d'une bibliothèque, on peut également se servir des normes émises par le Service des bibliothèques publiques quant au nombre de livres par habitant qu'une bibliothèque devrait posséder. Le tableau 3 illustre cette question ${ }^{8}$.

Tableau 3:

Bibliothèques publiques et nombre de livres par habitant

\begin{tabular}{l|c|c|c|cc} 
& $\begin{array}{c}\text { Jusqu'à } \\
9,999 \\
\text { habitants }\end{array}$ & $\begin{array}{c}\text { De } \\
10,000 \text { à } \\
24,999\end{array}$ & $\begin{array}{c}\text { De } \\
25,000 \text { à } \\
49,999\end{array}$ & $\begin{array}{c}\text { De } \\
50,000 \text { à } \\
199,000\end{array}$ & $\begin{array}{c}200,000 \\
\text { et } \\
\text { plus }\end{array}$ \\
$\begin{array}{l}\text { Normes pour } \\
\text { le nombre de } \\
\text { livres/tête }\end{array}$ & $3^{1} / 2-3^{1} / 4$ & $3^{1} / 4-3$ & $3-2^{1 / 2}$ & $21 / 2-2$ & 2 \\
$\begin{array}{l}\text { Nombre de } \\
\text { livres/tête }\end{array}$ & 2.55 & 2.14 & 1.25 & \multicolumn{2}{|c}{1.09} \\
$\begin{array}{l}\text { Nombre de bi- } \\
\text { bliothèques }\end{array}$ & 27 & 25 & 20 & \multicolumn{2}{|c}{17} \\
$\begin{array}{l}\text { Nombre de bi- } \\
\text { bliothèques } \\
\text { dépassant les }\end{array}$ & 11 & 4 & 1 &
\end{tabular}

Seulement 18 bibliothèques sur 89 (soit 20\%) dépassent le nombre requis de livres pour servir adéquatement leur clientèle. Et plus de la moitié de celles-ci se retrouvent dans les municipalités de moins de 9,999 habitants et surtout de moins de 5,000 habitants. En fait, l'importance numérique de la collection est inversement proportionnelle à l'importance numérique de la population. Aussi surprenant que cela puisse paraître, les petites municipalités ont des collections plus importantes que les grandes. Les statistiques restent, hélas, silencieuses sur la qualité de ces mêmes collections.

En tête du peloton, on retrouve, dans l'ordre, Notre-Dame-desMonts (22.86), Lac Brôme (6.61), Westmount (5.11), Ville Mont-Royal (4.98), Gagnon (4.63), Malartic (4.52), Amqui (4.34) et Pointe-Claire $(4.27)^{10}$.

8. Normes pour les bibliothèques municipales, Québec, Ministère des Affaires culturelles, Service des bibliothèques publiques, 1974, $62 \mathrm{p}$.

9. Statistiques pour l'année 1978 tirées de Biblio-contact, vol. 5, no 1 (printemps 1979).

10. Notre-Dame-des-Monts, Lac Brôme, Gagnon et Amqui ont moins de 5,000 habitants. 
La norme émise, qui varie de 2 à $31 / 2$ livres par habitant, permet à une bibliothèque de bien fonctionner. Avoir moins d'un livre par habitant, c'est se situer sous le seuil de l'indigence chronique. Comment peut-on envisager de servir adéquatement 50,000 habitants quand on dispose d'à peine 25,000 livres? Poser la question, n'est-ce pas y répondre du même coup? Pourtant 19 municipalités (21\%), dont 9 de plus de 50,000 habitants, se retrouvent dans cette situation. En 1979, les 89 bibliothèques publiques subventionnées offrent en moyenne 1.3 livres par habitant desservi.

\section{Dépenses par habitant pour l'achat exclusif de livres}

A ce chapitre, ce sont d'abord des bibliothèques de municipalités à population majoritairement anglophone qui, d'après les prévisions budgétaires $1979^{11}$, consacrent les sommes les plus considérables à l'achat de livres.

$\begin{array}{lrll}\text { Pointe-Claire: } & 113,000 \$ \div 25,900 \text { habitants } & =4.36 \$ \\ \text { Ville Mont-Royal: } & 65,000 \$ \div 20,500 " & =3.17 \$ \\ \text { Côte St-Luc: } & 71,855 \$ \div 25,700 \quad " & =2.80 \$ \\ \text { Montréal-Est: } & 18,150 \$ \div 4,186 " & =4.33 \$ \\ \text { Marieville: } & 11,450 \$ \div 4,853 \quad " & =2.36 \$^{12}\end{array}$

Si l'on considère que les bibliothèques qui desservent une population majoritairement anglophone achètent des livres anglais dans une proportion allant jusqu'à $80 \%$ et que le livre anglais coûte au moins deux fois moins cher qu'un livre de langue française et si on compare le prix d'un nouveau et bon roman en "pocket book» de langue anglaise, $1.95 \$, 2.95 \$, 3.95 \$$ avec celui d'un nouveau et bon roman de langue française en édition normale, à plus de $10 \$$, l'édition en livre de poche prenant toujours au moins six mois à paraître, l'écart apparaît dramatiquement démesuré.

II serait possible de combler le fossé. II faudrait que chaque bibliothèque desservant une population francophone consacre un minimum de $2 \$$ par habitant exclusivement pour l'achat de livres. Or, actuellement, l'ensemble des 89 bibliothèques n'y consacre en moyenne que $1.19 \$$ (prix de la reliure inclus). Quand on estime le prix moyen d'un livre non relié à environ $9 \$$ et si on compare ce prix à celui d'un paquet de cigarettes, il n'est pas du tout exagéré de demander à un Conseil municipal de consacrer $2 \$$ et plus par citoyen à l'achat des livres. C'est tout simplement demander non pas à vivre, mais à survivre. Pourtant, 37 bibliothèques du Québec (42\%) consacrent moins de $1 \$$ par habitant à l'achat de livres.

C'est à ce niveau fondamental qu'il faut faire débloquer les crédits. Si la bibliothèque publique ne peut offrir les nouveautés rapidement et en quantité, elle ne peut plus que prétendre jouer un certain rôle de conservation, elle est sans cesse en retard d'au moins une génération et elle gardera toujours son air arriéré, ennuyeux et poussiéreux. 


\section{Pourcentage du budget consacré à l'achat de livres}

A quelques exceptions près, le budget d'achat de livres d'une bibliothèque publique devrait représenter un minimum de 25 à $30 \%$ du budget total de fonctionnement pour réussir à rejoindre les normes. $\mathrm{Si}$ l'on exclut les bibliothèques en formation, onze bibliothèques consacrent plus de $25 \%$ de leur budget à l'achat de livres en 1979; parmi celles-ci, six desservent une population de moins de 5,000 habitants et consacrent des sommes par ailleurs peu élevées aux salaires.

Dans leurs prévisions budgétaires 1979, les 89 bibliothèques publiques prévoyaient affecter $19.6 \%$ de leur budget à l'achat de livres alors que les B.C.P. prévoyaient $28.4 \%$. D'ailleurs, en moins de dix ans, les directeurs des B.C.P. ont réussi à offrir 1.61 livres par tête comparativement à 1.30 pour l'ensemble des 89 bibliothèques municipales. II faut reconnaître toutefois que les B.C.P. jouissent de l'aide de nombreux bénévoles dans l'accomplissement de leur travail.

\section{Prêts par habitant}

Un des moyens de mesurer l'efficacité et la rentabilité d'une bibliothèque consiste à vérifier le nombre de prêts par habitant dans la municipalité. On peut dire qu'une bibliothèque qui ne prête pas au moins trois livres par habitant rencontre des difficultés dont la nature, pour être identifiée, exige une analyse spécifique, plus particulièrement dans le cas des municipalités de plus de 50,000 habitants. Dix des dix-sept municipalités de 50,000 habitants et plus ne font pas trois prêts par habitant.

La moyenne provinciale est de 2.7 livres prêtés par habitant en 1979 (la moyenne canadienne est de 4.9 en 1976). Le tableau 4 présente la situation générale en 1978.

\section{Tableau 4:}

\section{Répartition des bibliothèques selon les prêts par habitant}

$34(38 \%)$ ont entre 0 et 2.9 prêts

$34(38 \%)$ ont entre 3.0 et 4.9 prêts

$16(18 \%)$ ont entre 5 et 9.9 prêts

5 ( $6 \%)$ ont plus de 10 prêts: Pointe-Claire, 14.

Westmount, 13.8

Malartic, $\quad 11.8$

Gagnon, $\quad 10.9$

] $34(38 \%)$

Mont-Laurier, 10

\section{Quelques difficultés}

Ces quelques statistiques indiquent une situation très intéressante en certains endroits, mais surtout des lacunes à combler. II y a par ailleurs d'autres aspects de ce tableau qui viennent compliquer la bonne gestion d'une bibliothèque publique. Parmi ceux-ci, nous avons retenu, à titre d'exemples, les difficultés face aux élus municipaux et le statut des bibliothèques publiques. 


\section{Difficultés face aux élus municipaux}

Dans l'esprit de plusieurs élus municipaux, les livres et la bibliothèque sont importants... à long terme. A ce niveau, ils reconnaissent que la lecture permet le mieux-être, la découverte, l'invention, la culture, le développement. A court terme cependant, ils le contestent. Une bibliothèque n'est pas rentable, disent-ils. Elle ne rapporte pas. Le sort des bibliothèques est ainsi vite réglé, sans que ces mêmes personnes ne se soient par ailleurs demandées comment on mesure la rentabilité d'un service de police ou d'un aréna. D'ailleurs, au Québec, cette constatation pourrait s'appliquer à l'ensemble des personnes qui ont à prendre des décisions concernant les bibliothèques, à divers paliers administratifs (dans les hôpitaux, les universités, les écoles, etc.).

L'heureuse participation de la section des bibliothèques publiques de l'ASTED aux quatre derniers congrès annuels de l'Union des municipalités du Québec commence à porter ses fruits: les bibliothécaires prennent ainsi contact directement avec ceux qui ont l'autorité et le pouvoir de changer les choses.

Les espoirs de changement chez nos élus nous viennent actuellement des nouvelles villes champignons, villes de banlieue autour des grandes agglomérations du Québec et en particulier autour de Montréal. C'est là que les administrateurs municipaux et les citoyens, plus jeunes, plus éduqués, sont aussi plus ouverts aux nouvelles conceptions de la vie moderne, au changement et au besoin de savoir et d'information. Plusieurs de ces villes se sont dotées de bibliothèques avec des moyens financiers relativement suffisants.

Des problèmes demeurent: Québec, la capitale, commence à bouger, mais sa bibliothèque n'est pas un service municipal; elle demeure une corporation privée. Quant à Montréal, on tente d'y faire des miracles avec des miettes. Pourtant, la Ville de Montréal est fière de l'égalité salariale de ses policiers avec ceux de Toronto. A quand l'égalité des budgets consacrés aux bibliothèques à Montréal et à Toronto?

Or, la situation dans les deux grandes villes du Québec, Montréal et Québec, crée un problème pour les bibliothèques des autres municipalités. Elles ne sont pas le pôle de référence positif auquel les autres bibliothèques municipales seraient en droit de s'attendre. Comme tous les milieux, celui-ci a ses codes et ses modes. Les membres des conseils municipaux et les gérants des villes communiquent entre eux et plusieurs prennent (ou ont pris) Montréal comme exemple. Si la mode était aux bibliothèques à Montréal, plusieurs autres municipalités emboîteraient le pas. Mais c'est loin d'être le cas. Les bibliothèques de plusieurs municipalités se ressentent de cet état de fait de la même manière que l'économie générale du Québec se ressent de l'inertie ou de l'expansion économique de Montréal ${ }^{13}$.

13. A propos de Montréal et pour l'ensemble des bibliothèques publiques de la région de Montréal, voir C. Aubry et L.-G. Denis, Rapport de l'étude des bibliothèques publiques de la région de Montréal. Québec, Ministère des Affaires culturelles, Service des bibliothèques publiques, 1976, XVI-280 p. 


\section{Difficultés face au statut des bibliothèques publiques}

Dans l'esprit du public et des édiles municipaux, l'idée que n'importe qui peut s'occuper d'une bibliothèque est encore répandue. II en découle une sorte d'anarchie administrative, un laisser-faire général qui est à la fois la cause et la conséquence de l'anémie des bibliothèques publiques.

Au Québec, près de la moitié des bibliothèques municipales ne constituent pas un service municipal autonome égal hiérarchiquement aux services de la police, des loisirs, de l'évaluation, des finances: on les retrouve comme sous-service du service des loisirs, quand ce n'est comme sous-sous-service. On a même vu des bibliothèques qui ne sont pas administrées par un bibliothécaire.

De plus, au sein même du ministère des Affaires culturelles, le Service des bibliothèques publiques, avec la plus grosse enveloppe budgétaire de ce ministère, se retrouve au plus bas de la hiérarchie administrative. La volonté exprimée d'accorder la priorité aux bibliothèques publiques n'a aucun écho au niveau des structures gouvernementales et le Gouvernement, du moins pour le moment, n'entend pas exiger l'autonomie du service de la bibliothèque à l'intérieur de la hiérarchie municipale comme condition aux subventions.

Tout ceci contribue à accentuer l'isolement de plusieurs collègues, à maintenir sous-développées ces bibliothèques, ce qui a un effet d'entraînement négatif sur le développement des autres. II est communément entendu que la place dans une hiérarchie détermine la place dans les priorités et... les budgets. Dépendre d'un autre service, c'est dépendre des priorités de ce service, avec tout ce que cela implique. C'est surtout n'avoir aucun accès aux sources du pouvoir municipal, c'est-à-dire au gérant municipal et, en dernier ressort, au Conseil municipal et au Maire. C'est souvent la marginalisation.

Avoir accès directement au pouvoir avec le moins d'intermédiaires possible, c'est déjà avoir un statut reconnu, c'est déjà un meilleur budget, c'est être dans le bon circuit, c'est être ou pouvoir se rendre présent dans les préoccupations des élus et des citoyens. En fait, ceci n'est pas propre aux bibliothèques publiques. Plus la place d'une bibliothèque (universitaire, de collège, d'entreprise, etc.) est élevée dans une hiérarchie administrative, plus ses chances de succès sont augmentées.

Pour les bibliothécaires-directeurs d'un service autonome de bibliothèque au sein de l'administration municipale, il y a autant de différence entre un service de bibliothèque et un service des loisirs qu'entre un service de bibliothèque et un service de police.

Un service de bibliothèque autonome doit exister dans chaque municipalité. II a des buts, des politiques et des objectifs qui lui sont propres, qui ont leur propre dynamique et qui exigent un fonctionnement particulier sans rapport et parfois même diamétralement opposé à celui d'un autre service. 


\section{Conclusion}

L'amélioration du réseau des bibliothèques publiques pose aux professionnels du milieu de multiples exigences de compétence, de dynamisme, d'innovation et de créativité. Elle exige même, à certains moments, des talents de pionniers. Mais elle représente toujours des défis administratifs emballants.

II faut surtout que les bibliothécaires des bibliothèques publiques soient des vendeurs, d'excellents vendeurs... d'un produit aussi essentiel que l'eau et le pain. Ils doivent vendre, solliciter... sinon hurler aux élus du peuple et aux six millions de Québécois qu'une bibliothèque n'est pas une faveur, une récompense, mais un droit que tout citoyen peut et doit réclamer, un devoir que toute municipalité doit remplir. Ils doivent surtout démontrer qu'en 1979, il y a un minimum vital au-dessous duquel il est impossible de vivre.

Vendre les bibliothèques publiques, c'est expliquer aux élus et à la population ce qu'est une bibliothèque publique, ce à quoi elle répond, ce qu'elle demande comme exigences, normes, minimum. Cela implique la traduction du jargon bibliothéconomique en termes administratifs clairs et précis, une vulgarisation accessible à la compréhension des citoyens et des élus. Quant au reste, le pouvoir municipal compte sur les bibliothécaires professionnels pour accomplir et régler les problèmes particuliers et spécifiques à l'exercice de la profession.

Pour vendre avec efficacité, il faut aussi un minimum de concertation, de coordination et d'appui des milieux intéressés. L'aide financière est un aspect primordial. Mais il y a plus. II faut souhaiter la multiplication de certaines initiatives de la part du ministère des Affaires culturelles, comme cet atelier d'information tenu au congrès de I'Union des municipalités du Québec, en septembre 1979, à Québec. Tout en respectant l'autonomie municipale, de telles rencontres avec les responsables municipaux permettront de les sensibiliser aux problèmes de la lecture, à leurs obligations, de fixer des objectifs budgétaires, des programmes, des échéances et peut-être, qui sait, de susciter une certaine concertation des citoyens pour une politique de la lecture s'adressant aux six millions de citoyens québécois. Si une telle concertation a été possible entre libraires, éditeurs et distributeurs, pourquoi ne le serait-elle pas avec les autorités municipales?

Simultanément à ces efforts constants, il est nécessaire et urgent de prévoir une campagne de publicité sur le droit des Québécois à des bibliothèques publiques. Cette campagne gouvernementale devrait être conçue dans le même esprit que celles que l'on a pu voir et que l'on voit encore annonçant le projet de loi 24 (droit des enfants), l'assurance-automobile, ...et une panoplie de services publics. II faut absolument sensibiliser la population à ce produit gratuit, la bibliothèque publique, produit qui peut se consommer aussi facilement que bière et hamburgers.

Lors de la rencontre avec les membres de la Commission des bibliothèques publiques le 16 février 1979, les huit directeurs invités à conseiller cette Commission ont émis le souhait urgent 
d'avoir des spécialistes pour mettre sur pied une campagne de publicité structurée basée sur les groupes-cibles, les produits offerts, les ressources disponibles.

Une telle campagne favoriserait l'utilisation des bibliothèques existantes, inciterait les citoyens qui habitent dans des villes n'ayant pas de bibliothèque municipale à en réclamer une au même titre qu'un centre sportif ou un terrain de baseball. Toutes les bibliothèques existantes en retireraient des bénéfices. Chaque bibliothèque pourrait compléter le travail amorcé selon sa spécialité. Le Québec investit des ressources financières importantes dans les bibliothèques publiques: il devrait en faire la promotion, pour améliorer le retour sur son investissement.

Pour mieux se vendre auprès des contribuables québécois, les bibliothèques publiques - surtout celles à créer - devraient pouvoir se départir de leurs services techniques, beaucoup trop prohibitifs pour chacune. Une centralisation est indispensable à plus ou moins court terme. Les bibliothèques publiques doivent être le plus près possible de leurs clients, leur seule raison d'être, et laisser à d'autres organismes, régionaux ou autres, le soin des travaux techniques. Ne pourrait-on pas imaginer un contrat entre le ministère des Affaires culturelles et la Centrale des bibliothèques qui confierait à cette dernière le traitement, dès leur parution, de tous les livres susceptibles d'intéresser les bibliothèques publiques et l'envoi gratuit et hebdomadaire des fiches correspondantes!

Si, jusqu'à tout récemment, les structures mentales, sociales, économiques et politiques n'ont pas favorisé l'essor des bibliothèques publiques québécoises, on remarque depuis plusieurs années que des efforts sont en cours, des crédits débloqués, des initiatives prises, des expériences tentées... II faut emboîter le pas et vouloir, quinze ans après la révolution tranquille et les changements dans notre système d'éducation, mettre les bibliothèques publiques sur la carte du Québec.

Le ministère des Affaires culturelles a raison d'accorder priorité aux bibliothèques publiques. Peut-on espérer que le Gouvernement fasse publiquement sienne et accorde un appui sans réserve à cette priorité du ministère des Affaires culturelles? Le Québec de demain sera aussi à l'image de "Dis-moi ce que tu lis, je te dirai ce que tu es". 